\title{
High Reproducible Scanning Near-field Optical Microscopy with a Few Nanometer Lateral Spatial Resolution.
}

\author{
Takehiro Tachizaki ${ }^{1}$, Keifeng Zhang ${ }^{2}$, Ichiro Yamakawa ${ }^{2}$, and Shin-ichi Taniguchi ${ }^{2}$ \\ 1. School of Engineering, Tokai University, Hiratsuka, Japan. \\ 2. Research \& Development Group, Center for Technology Innovation - Production Engineering, Hitachi, \\ Ltd., Yokohama, Japan.
}

Scanning near-field optical microscopes (SNOM) have enabled sub-diffraction limit optical imaging and measurement [1]. A number of research and development activities on SNOM and related technique realized spectroscopic measurement of a few nanometer lateral spatial resolution with nondestructive and noninvasive method [2]. Practicability of sub-diffraction resolution and spectroscopic measurement made SNOM a major tool in various scientific fields. However, SNOM has an awaiting solution: measurement reproducibility under a few nanometer resolution.

Low reproducibility of SNOM is due to the mechanical properties of its probes. Probes of SNOM are, conventionally, prepared by sharpened silica fiber coated with thin metal film or etched metal needle [1, 2]. The mechanical properties of these materials such as Young's modulus determine the endurance of probes. Enhancing the rigidity of probes realize higher reproducible measurement with high spatial resolution.

In this work, we applied multiwall carbon nanotube (MWNT) to optical probe for enhancing the endurance. MWNT was attached at the top of silicon tip which is used for the probe of atomic force microscope [3]. Propagating laser beam focused on the edge of the metal coated silicon tip generated surface plasmon polariton which transfer the optical energy to the top of the silicon tip. Near-field to near-field coupling technique transferred optical energy from the top of the silicon tip to the apex of the MWNT, and generated a nanometric optical spot at the apex of the optical probe. Contact with the sample surface scattered near-field from the apex of the probe. Conventional theory of light scattering teaches the scattering potential of the sample surface decide the intensity of scattered light [4]. By monitoring scattered light intensity, we are able to obtain the refractive index or dielectric constant of the sample surface.

In experiments, we modified a commercialized atomic force microscope for optical measurement by adding optics in order to excite near-filed and detect the scattered light. Intensity of the scattered light was recorded as a function of probe position to obtain the two dimensional map of refractive index. We used nanometer-sized surface structure for calibration sample. Flat surface with nanometer width patterns of metal and silica was fabricated by thin film deposition techniques and surface polishing technique.

Figure 1 (a) shows an example of maps of scattered light intensity. This map indicates that our novel optical measurement technique is able to catch the spatial change of surface refractive index with a few nanometer lateral spatial resolution. Together with surface topography which was obtained at the same position and same time, sectional profile of scattering light intensity is shown in Fig. 1 (b). Signal variation of scattered light intensity (Fig. 1 (b) solid line) indicates the optical measurement performed in this work catches the position of material interfaces clearly. 
In order to estimate the optical resolution, we performed three dimensional finite-difference timedomain (FDTD) numerical simulations. Figure 2 (a) and (b) show simulated optical spot distribution and signal variation around metal line of $7 \mathrm{~nm}$ width, respectively. FDTD simulations indicate spot radius at 1/e intensity corresponds to the width of scattered light intensity form $20 \%$ to $80 \%$. Supposing the spatial resolution is defined by the optical spot radius, the spatial resolution of our experiment was 3.8 nm.

We also estimated the reproducibility by means of a statistical method. The distance of peak structures in scattered light intensity at the both interface of metal line (pointed by triangle in Fig.1 (b)) were used to determine the position accuracy. From the experimental results shown in Fig. 1 (a), dozens of intervals of peaks were collected. The distribution of these intervals, on assumption the Gaussian distribution, shows standard deviation of $2.5 \mathrm{~nm}$. This high reproducibility coexistence with a few nanometer spatial resolution is promising for industrial application of SNOM.

[1] E. Betzig, et al., Science 251 (1991) p. 1468.

[2] S. Kawata, Y. Inouye, and P. Verma, Nature Photonics 3 (2009), p. 388.

[3] H. Dai, et al., Nature 384 (1996), p. 147.

[4] M. Born and E. Wolf, "Principles of Optics" (Cambridge University Press, 1999).
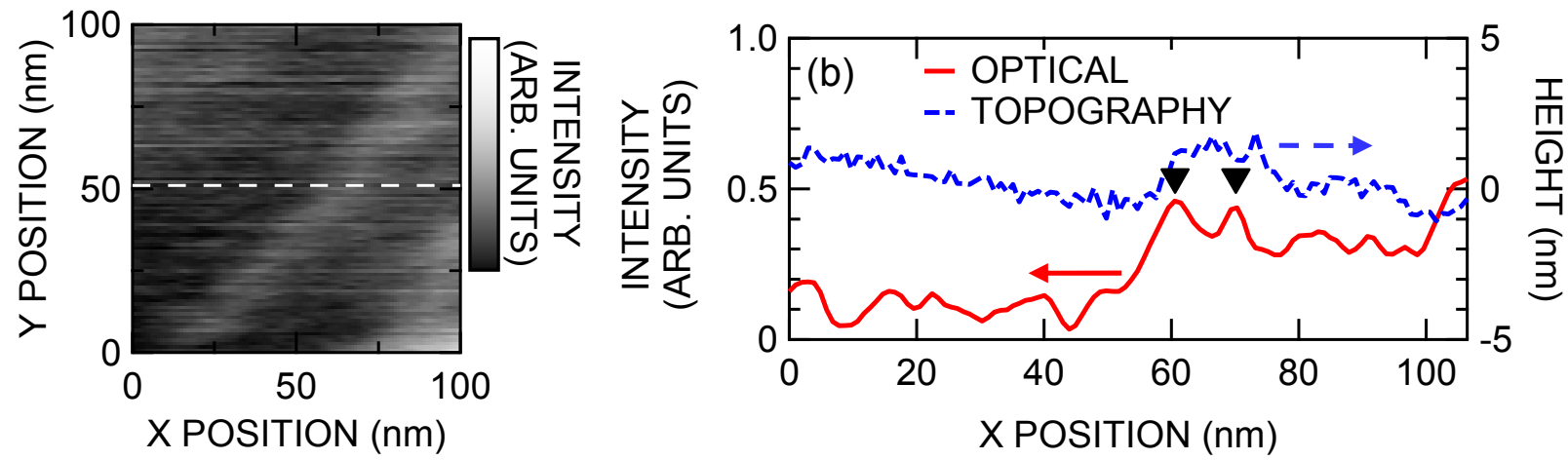

Figure 1. Experimental results. (a) Two dimensional map of scattered light intensity. Solid and dashed lines in (b) represent optical signal variation and surface topography at the dashed line in (a), respectively. Peaks pointed by triangles in (b) indicate places of material interfaces.

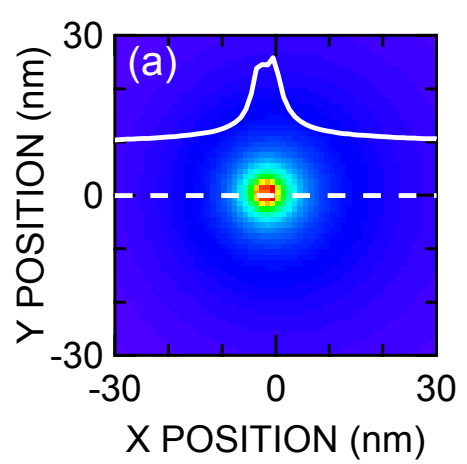

(b)

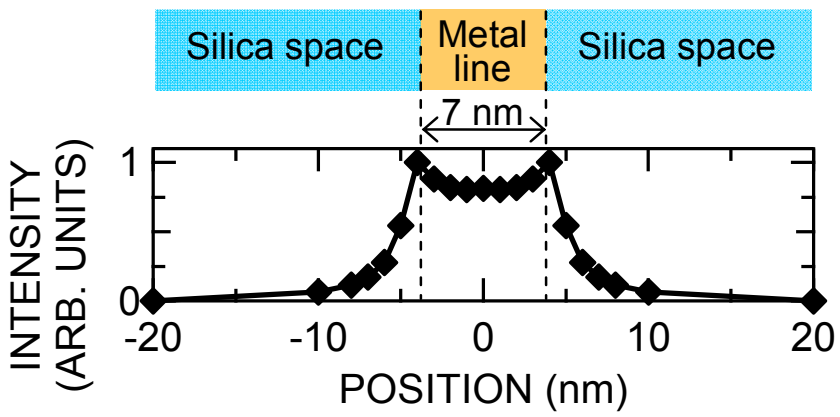

Figure 2. FDTD simulation results. (a) Two dimensional contour plot and sectional profile of the spot at the apex of optical probe. Upper panel of (b) is a schematic sample structure. Lower panel of (b) shows change of optical intensity as a function of the probe position. 